\title{
Effective treatment of necrobiosis lipoidica using intense pulsed light therapy
}

Low-risk method presents quick turnaround of the degenerative disease, researchers found

A patient with chronic degenerative disease of the collagen, after two years of unsuccessful treatment, underwent intense pulsed light (IPL) therapy for 14 months and obtained an excellent result, reported a team of Brazilian dermatologists.

"The successful outcome of this case report suggested that IPL may represent a worthwhile option in the treatment of NL, offering low risks of collateral effects, especially when administered by experienced professionals," wrote Dr. Marisa Gonzaga da Cunha and her co-authors from São Paulo's Faculty of Medicine of ABC and Federal University of São Paulo, Brazil, in a case study published by Journal of Surgical Dermatology (JSD).

The patient was afflicted with necrobiosis lipoidica (NL), a disorder of collagen degeneration that typically manifests as one or more tender yellowish-brown patches on the lower legs, usually the shin, developing slowly over several months or even persisting for years. At the center of the patch is usually a shiny, pale area showing prominent blood vessels; and while NL is usually painless, it could ulcerate upon minor injuries with a risk of secondary bacterial infection and delayed healing.

Necrobiosis lipoidica affects people of all races and occurs at any age, but it is three times more common in women than in men, according to the British Association of Dermatologists. The cause of necrobiosis lipoidica has not yet been fully understood by modern science, and it has been surmised that NL may have followed damages to the collagen, or fibers which give the skin its strength, possibly "due to changes in the small blood vessels of the skin," the association stated.

While NL is not contagious or cancerous, "there is a small risk of skin cancer (squamous cell carcinoma) developing in longstanding lesions," added the British Association of Dermatologists. The condition, when first described by dermatologists in 1920s, was postulated to be in conjunction with diabetes. It was not until in the 1960s when the disease, originally named 'dermatitis atrophicans lipoidica diabetic' was relabeled as necrobiosis lipoidica, omitting altogether of whether the patient is diabetic or not.

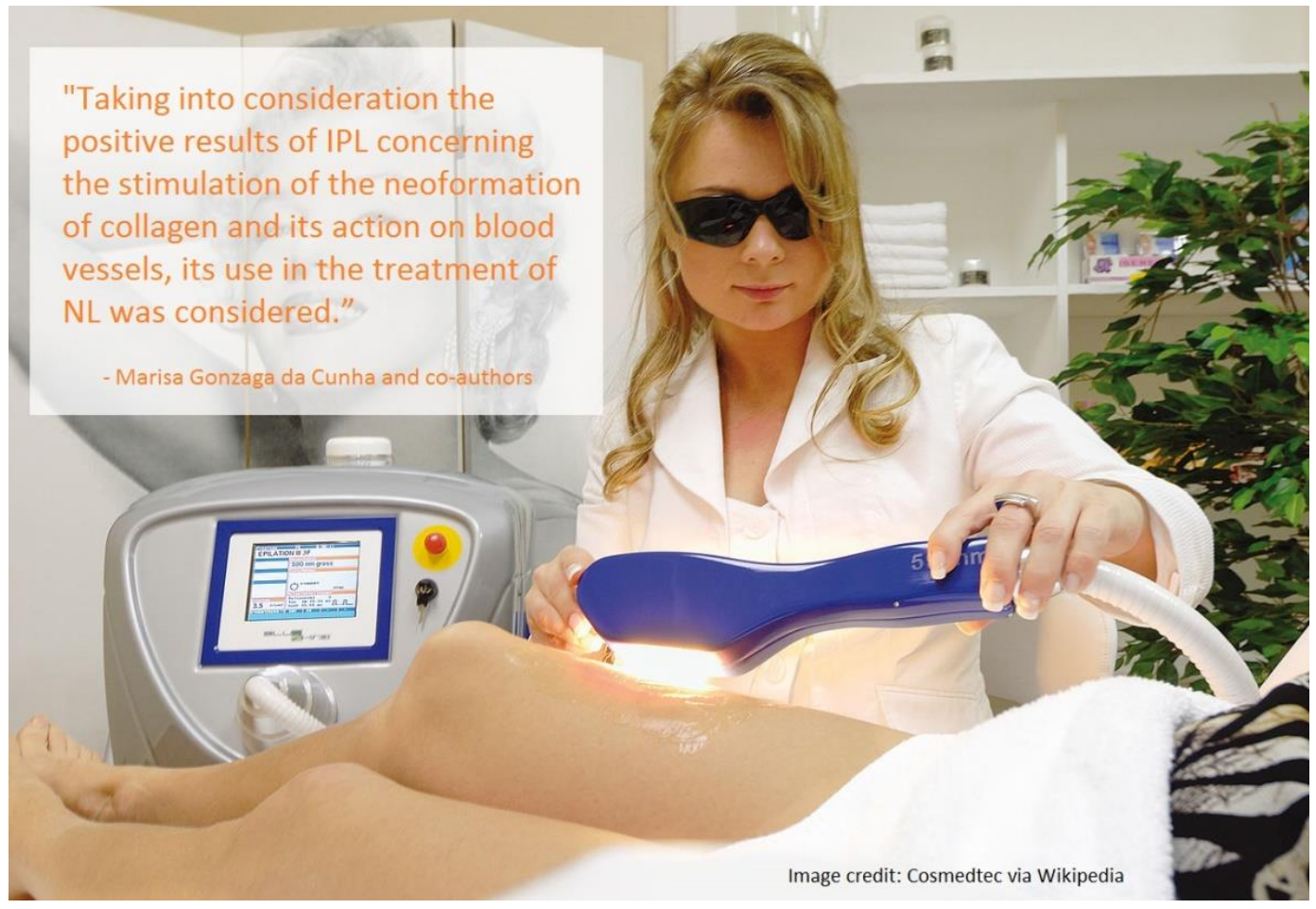




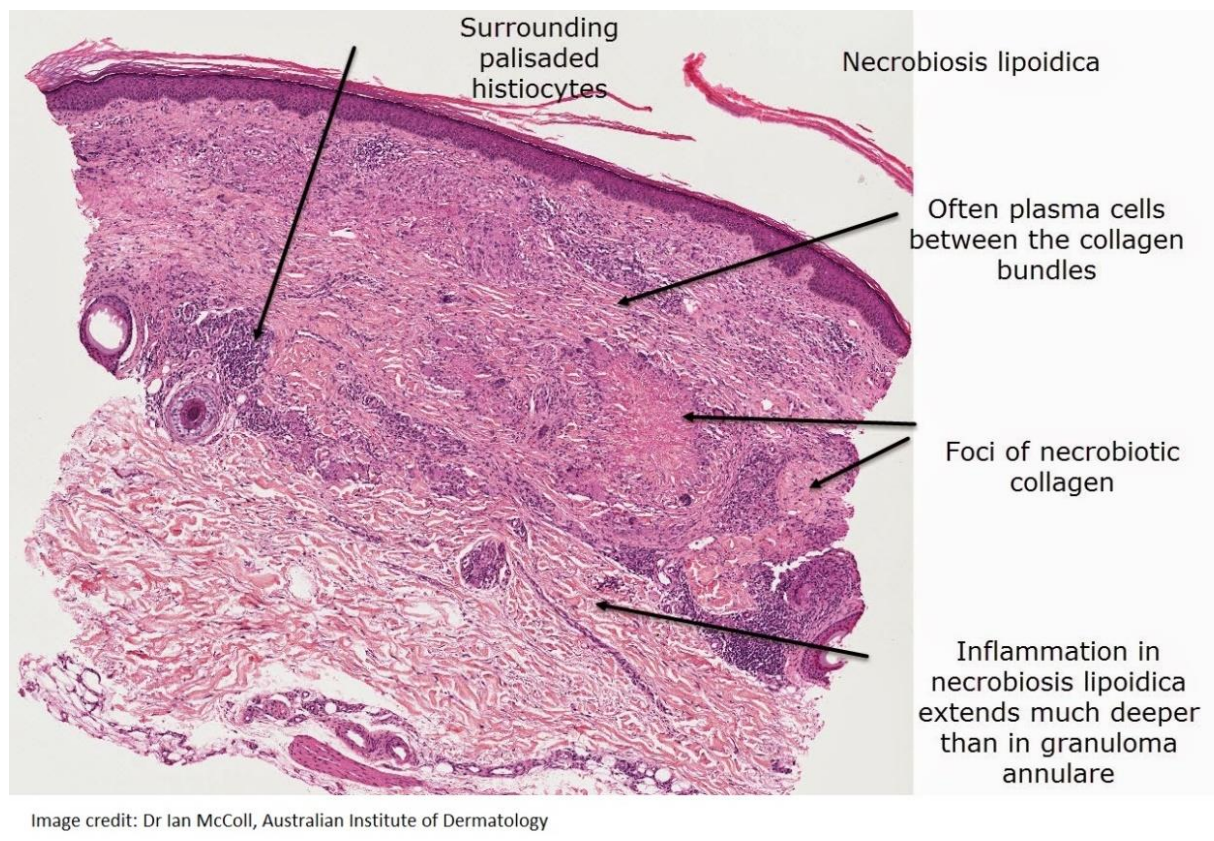

The link between NL and diabetes has still not been clearly determined yet, and the hypotheses proposed range from an abnormality in glucose transport to the degradation of the collagen. Nevertheless, according to the British Association of Dermatologists, while one in 300 diabetics has NL, "most patients with necrobiosis lipoidica have, or will develop, diabetes."

There have been a number of proposed treatments of NL, "but none is $100 \%$ effective," Dr. da Cunha and co-authors said, adding that the therapies often resulted in varied responses, "typically with flattening of borders and almost always a partial clearing with persistent central atrophy."

On the other hand, the researchers noted that intense pulsed light has already been a staple treatment by dermatologists for conditions such as acne vulgaris, vascular and pigmentary disorders, photo-aged skin and scars. "Its basic principle consists of selective photothermolysis - in other words, light energy is absorbed by a specific target chromophore with energy transfer and heat generation, which promotes its subsequent modification. Wavelength can be established according to the absorption peak dependence of the target chromophore. Pulse duration should last less than the thermal relaxation time, which limits heat diffusion and lesion to nearby structures," their report stated.

With the aid of its cut-off filters, the main advantage of an IPL device is its versatility of wavelengths, fluencies, pulse duration and intervals, which allows for the treatment

of a great variety of lesions, the Brazilian researchers said. "It is possible to adjust them according to the type, depth and size of the lesion as well as the patient's skin type so that maximum improvement can be achieved without epidermal loss or collateral effects," they added.

Hence, "[t]aking into consideration the positive results of IPL concerning the stimulation of the neoformation of collagen and its action on blood vessels, its use in the treatment of NL was considered," said the authors.

The female patient in their case study was treated with IPL monthly for a period of 14 months, and rapid improvements in her condition were observed beginning from the second session. After 13 sessions, her skin quality improved significantly, displaying fewer blood vessels and with the edges of the redness of the skin returning to a tone similar the surrounding area, the authors described.

The result confirmed the researchers' conjecture that IPL promotes tissue remodeling through the formation of new dermal collagen, the increased activity of fibroblasts (which produces collagen), and the decrease in content and rearrangement of the highly elastic elastin fibers.

"As IPL stimulates neocollagenesis, it was selected due to its use as a therapeutic attempt, resulting in an excellent response," they reported.

Marisa Gonzaga da Cunha, Flávia Cury Rezende, Ana Lucia Gonzaga da Cunha and Carlos D'Apparecida Santos Machado Filho are co-authors of this featured case report which is published on pages 31-34 in this JSD issue. 